\title{
Buchanzeige
}

Sehwarz, 0., Die Funktionsprüfung des Auges und ilire Verwertung fiir die allgemeine Diagnostik. Fíir Arzfe und Studierende. •Berlin, S. Karger. 1904. 322 Seiten mit 81 Figuren im Text und einer ïafel.

Bei der Abfassung dieses Buches hat dessen Autor sich die Aufgabe gestellt, nicht nur eine ausfiihrlichere Darstellung der Methoden der Funktionsprüfung zu geben, sondern im Zusammenhang damit auch die Funktions-störungen selbst und deren nächste Ursachen in der Weise darzustellen, wie sie sich der Untersuchende selbst, wenn er zielbewusst arbeiten will, vor Augen balten muss. Hierbei wurdo ganz besonders auf die Beziehung der Augenkrankheiten zudeninneren Krankheiten, speziell zu den Krankheiten des Centralnervensystems Rûcksicht genommea. Man kann sagen, dass der Ver-fasser díeser Aufgabe gerecht geworden ist. Der Anfänger wird in dem Werke einen sicheren Führer finden, den Spezalisten aber $\Lambda$ vird manche Be-obachtung, mancheneue Auffassung, man cheneueDars tell ungs weise interessieren. Als Grnndlage zum Verständnis der Funktionsprüfungen werden im 1. ïeil die Grundzüge der Optik des Auges behandelt. So gut und klar dieser Teil auch abgefasst ist, so ware doch wohl eine einfachere Art der Darstellung möglich, wenn es sich bloss darnm handelt, die für die praktische Ausführung der Funktionsprüfung notwendigen Grundbegriffe darzulegen· Jenem Leser aber, der sich auch tiefergehende Kenntnisse verschaffen will, wird der Stoff in sehr anregender Form geboten. In ungewöhnlícher, aber sehr guter Art wird die Lehre vom Astigmatismus vorgeführt. Bei der Untersuchung der Brillengläser ware ein Hinweis auf den praktischen ameri-kanischen Linsenmesser $\Lambda$ vünschenswert. Die nur als Ergänzung angéiügte, $\lambda$ vichtige Formel des Objekt- und Bildabstandes von den Hauptbrennpunkten wird wohl in einer 2. Auflage an der passenden Stelle in den Text ein-gefügt werden.

Der 2. Teil ist der eigentlichen Funktionsprüfung gewidmet. Er zer-fällt in 3 Abschnitte, von denen der erste unter dem Titel „Leistungen der Wahrnehmung” eine Darstellung der Untersuchung der Refraktions- und Akkomodationsanomalien, der Sehschärfe, des Licht- und Farbensinnes, der Gesichtsfelduntersuchung, sowde deren Anomalien enthält. In dem Kapitel über die Befraktionsanomalien sind auch die neuesten TJntersuchungen, speziell diejenigen von Hess, entsprechend berücksichtigt. Das Kapitel über die Pruning des Farbensinnes ist dagegen etwas zu kurz gehalten. In einem besonderen Kapitel am Schlusse dieses Abschnittes wird die „erkennende 284 Literatur-Verzeichnis.

Wahrnehmung" - die Verwertung der $\Lambda$ Vahrnehmungen durch das Seh-gedäclitnis - und als deren Stoning die Seelenblindheit besprochen.

Der 2. Hauptabschnitt enthält die Leistungen des Blendenapparates. Das hier gegebene Schema der Pupillenreaktíon lässt eine einfache Erklärung der einseitigen reflektorischen Pupillenstarre zu. $1 \mathrm{~m}$ 3. Hauptabschnitt des 2. Teiles werden die Leistungen des Bewegungsapparates und deren Unter-suchung vorgeführt. Die Darstellung dieser 2 Hauptabschnitte ist klar und vollständig, die Einteilung übersichtlich. In einem Anhange zum 2. Abschnitt, der sowohl die Augenmuskelstörungen als auch die Anomalien der Lid-muskulatur enthält, kommt die 
Simulation und deren Untersuchung zur Sprache. Bezüglich der Untersuchungsmethoden ware hier allerdings eine grössere Vollständigkeit am Platze, da die angeführten Methoden in die meisten Lehr-bücher aufgenommen sind.

Sehr erwünseht wird clem Leser der 3. Teil des Buches sein, in welehem in gedrängter Kürze eine Übersicht über die wichtigsten diagnostischen Schiüsse, die sich aus den durch die Funktíonsprüfung gewonnenen Befunden ergeben, geboten werden.

Das Buch kann alien Arzten, die sich eingehender mit der Ophthal-mologie befasseo wollen, bestens empfohlen werden, ebenso auch alien jenen, die sich mit interner Medizin, Neuropathologie oder Psychiatrie beschäftigen und die Funktionsprüfung des Auges von ihrem Standpunkte aus kennen lernen wollen. Gerade diese werden besonders den Teil des Buches, der auf die Besprechung der Ilefraktionsanomalien folgt, mit Vorteil studieren. So wird das Werk seiner ganzen Eigenart nach recht wohl neben der vor kurzem erschienenen Bearbeitung der Untersuchungsmethoden von Landolt seinen Platz behaupten, briugt ja diese letztere Arbeit, wie jetzt in der II. Auflage des Handbuches der Titel dieses Abschnittes richtig besagt, nicht allein eine Darstellung der Funktionsprüfungen. Gerade diese aber inter-messieren, wie oben angedeutet, viele $\Lambda$ rzte mit Recht ganz speziell.

Dimmer.

Literatur-Verzeichnis.

Anatomie. - Physiologic - Pathologie.

Ayres, The one-hand method of texting of the tension of the eye. Amer. Journ. of 0 phi halm. Januar.

Baas, K., Kristalldrusen in und unter der abgelösten Netzhaut. Arch. f. Ophthalm. 57. Bd. 3. H. Beaumont, W- M., Eye symptoms of rheumatoid arthritis. Ophthalm. soc. of London. 28. Januar. (Lancet. No. 4197.)

Bernstein, Felix, Das Leuchtturmphänomen und die scheinbare Form des Himmelsgewölbes. Zeitschr. f. Psychol. Bd. 34. H. 2. BorsckJce, Alfred, Untersuchungen über die Herabsetzung der Sehschärfe durch Blendung. Zeitschr. f. Psychol. Bd. 34. H. 2. BrovJcsbanJc-James, G. T., Asthenopia. Ophthalm. soc. of London. 28. Januar.

\section{(. (Lancet}

No. 4197.) Bultler

Karl

Beiträge zur Lehre von der Umstimmung des Sehorgans.

Diss. Freiburg i. Br. 